Proceeding Series of the Brazilian Society of Computational and Applied Mathe matics

\title{
Função Penalidade Baseada em Função Triangular para o Tratamento de Variáveis Discretas do Problema de Fluxo de Potência Ótimo
}

\section{Daisy Paes Silva ${ }^{1}$}

Faculdade de Engenharia, Pós-graduação em Engenharia Elétrica, Unesp, Bauru, SP

\section{Augusto Cesar Pereira ${ }^{2}$}

Faculdade de Engenharia, Engenharia Elétrica, Unesp, Bauru, SP

Edilaine Martins Soler ${ }^{3}$

Departamento de Matemática, Faculdade de Ciências, Unesp, Bauru, SP

Resumo. O problema de Fluxo de Potência Ótimo determina o estado de um sistema de transmissão de energia elétrica que otimize um dado desempenho e satisfaça restrições físicas e operacionais do mesmo. O problema é modelado como um problema de programação não linear com variáveis discretas e contínuas. Propõe-se um método de solução para este problema que trata as variáveis discretas por uma função penalidade obtida pela aproximação da função triangular por série de Fourier. O solver IPOPT é utilizado para resolver a sequência de problemas penalizados. As soluções dos problemas penalizados convergem para a solução original. Testes como sistema IEEE 14 barras são apresentados.

Palavras-chave. Fluxo de Potência Ótimo, Programação Não Linear, Variáveis Discretas, Função Penalidade.

\section{Introdução}

O problema de Fluxo de Potência Ótimo (FPO) é modelado por:

$$
\begin{aligned}
& \operatorname{Min} \mathrm{f}(x, y) \\
& \text { s.a: }\left\{\begin{array}{l}
h(x, y)=0 \\
g(x, y) \geq 0 \\
\bar{x}<x<\underline{x} \\
y_{i} \in D_{y_{i}}, i=1, \ldots, n_{y}
\end{array}\right.
\end{aligned}
$$

\footnotetext{
1 daisypaess@gmail.com

2 augustocp1993@gmail.com

3 edilaine@fc.unesp.br
} 
em que, $x$ são as variáveis contínuas: magnitude da tensão nas barras e ângulo da tensão, e $y$ são as variáveis discretas: tap dos transformadores e susceptâncias shunt; $D_{y_{i}}$ é o conjunto de valores discretos para $y_{i} ; f$ são as perdas de potência ativa nas linhas de transmissão; $h$ e $g$ são restrições físicas e operacionais do sistema: $h$ representa o balanço de potência ativa e reativa e $g$ representa os limites da geração de potência reativa injetada. A maioria das abordagens ignora a natureza discreta das variáveis e considera todas como contínuas. Essas abordagens não são realistas, pois algumas variáveis somente podem ser ajustadas por passos discretos. Uma função penalidade obtida pela aproximação da função triangular por série de Fourier para tratar das variáveis discretas do problema:

$$
\phi(\mathrm{y})=\frac{8}{225 \pi^{2}} 225 \operatorname{sen}\left[2 \pi\left(\frac{y}{s^{\text {sup }}-s^{\text {inf }}}+\alpha\right)\right]-25 \operatorname{sen}\left[6 \pi\left(\frac{y}{s^{\text {sup }}-s^{\text {inf }}}+\alpha\right)\right]+9 \operatorname{sen}\left[10 \pi\left(\frac{y}{s^{\text {sup }}-s^{\text {inf }}}+\alpha\right)\right]
$$

Para resolver o problema de FPO propõe-se resolver pelo solver IPOPT, problemas contínuos como dado, aumentando-se gradativamente o valor do parâmetro de penalidade:

$$
\begin{array}{r}
\text { Min } \mathrm{f}(x, y)+\gamma \phi(y) \\
\text { s.a: }\left\{\begin{array}{l}
h(x, y)=0 \\
g(x, y) \geq 0 \\
\underline{x}<x<\bar{x} \\
\underline{y}<y<\bar{y}
\end{array}\right.
\end{array}
$$

\section{Resultados e Considerações Finais}

Foram realizados testes com o sistema IEEE 14 barras. A solução ótima foi obtida na $7^{a}$ iteração em 5.608 segundos. A perda de potência ativa nas linhas nesta solução foi 12.32 MW. O método proposto mostrou-se eficiente para a resolução do problema de FPO para tal sistema. Futuramente, serão realizadas simulações com os sistemas IEEE 30, 118 e 300 barras para validar a eficiência da abordagem proposta.

\section{Agradecimentos}

A autora Daisy Paes Silva, bolsista CNPq - Brasil, agradece o apoio financeiro.

\section{Referências}

[1] E. M. Soler, E. N. Assada e G. R. M. Costa, Penalty-Based Nonlinear Solver for Optimal Reactive Power Dispatch With Discrete Controls. IEEE Transactions on Power Systems, [S.1.], vol. 28, 2174-2182, (2013).

[2] A. Wätchter e L. T. Biegler, On the implementation of an interior-point filter line-search algorithm for large-scale nonlinear programming. Mathematical Programming, vol 1, 2557, (2006). 